\title{
SOLUTIONS OF ABREU'S EQUATION WITH ROTATION INVARIANCE *
}

\author{
A. N. W. HONE ${ }^{\dagger}$
}

\begin{abstract}
We consider a fourth order nonlinear partial differential equation in $n$-dimensional space introduced by Abreu in the context of Kähler metrics on toric varieties. Rotation invariant similarity solutions, depending only on the radial coordinate in $\mathbb{R}^{n}$, are determined from the solutions of a second order ordinary differential equation (ODE), with a non-autonomous Lagrangian formulation. A local asymptotic analysis of solutions of the ODE in the neighbourhood of singular points is carried out, and the existence of a class of solutions on an interval of the positive real semi-axis is proved using a nonlinear integral equation. The integrability (or otherwise) of Abreu's equation is discussed.
\end{abstract}

1. Introduction. In recent work Abreu has considered toric Kähler metrics on toric varieties [4] or toric orbifolds [5] of dimension 2n. Following a construction due to Guillemin [14], each such variety or orbifold is completely determined by its moment polytope $P$ in $\mathbb{R}^{n}$, and the scalar curvature $S$ of the Kähler metric is given by a formula

$$
S=-\partial_{j} \partial_{k} u^{j k}
$$

where the matrix elements $u^{j k}$ are functions of $\mathbf{x}=\left(x^{1}, \ldots, x^{n}\right)^{T} \in \mathbb{R}^{n}$, and $\partial_{j}$ denotes the partial derivative $\partial / \partial x^{j}$ (the summation convention is assumed). More precisely, in terms of a symplectic potential function $u(\mathbf{x})=u\left(x^{1}, \ldots, x^{n}\right)$ on $\mathbb{R}^{n}$, the metric on the interior of the polytope $P$ is

$$
d s^{2}=u_{j k} d x^{j} d x^{k},
$$

where $u_{j k}$ are the elements of the Hessian matrix $\mathbf{H}$, i.e.

$$
\mathbf{H}=\left(u_{j k}\right), \quad u_{j k}=\partial_{j} \partial_{k} u,
$$

and in (1.1) the curvature is determined in terms of the inverse matrix

$$
\mathbf{H}^{-1}=\left(u^{j k}\right) .
$$

The metric $d s^{2}$ on the polytope $P$ extends to a metric $g$ given by

$$
g=u_{j k} d x^{j} d x^{k}+u^{j k} d \theta_{j} d \theta_{k},
$$

where $x^{1}, \ldots, x^{n}$ and $\theta_{1} \ldots, \theta_{n}$ are symplectic coordinates on the toric variety, with curvature given by (1.1).

In [4] Abreu was concerned with so called extremal toric metrics (in the sense of Calabi), and it was shown that the condition for extremality is that the curvature is an affine function of $\mathbf{x}$, in other words

$$
\partial_{j} S=\text { constant }, \quad j=1, \ldots, n .
$$

\footnotetext{
*Received June 30, 2003; accepted for publication April 28, 2004.

${ }^{\dagger}$ Institute of Mathematics \& Statistics, University of Kent, Canterbury CT2 7NF, UK (anwh@kent.ac.uk).
} 
Abreu constructed such metrics from potentials of the form

$$
u=\frac{1}{2} \sum_{l=1}^{d} \ell_{l}(\mathbf{x}) \log \ell_{l}(\mathbf{x}),
$$

where the $\ell_{l}(\mathbf{x})$ are affine functions which determine the facets of the polytope by the equations $\ell_{l}(\mathbf{x})=0, l=1, \ldots, d$. For the simplest case of Guillemin's construction [14], the polytope is a cuboid in $\mathbb{R}^{n}$, so that $d=2 n$, and the toric variety is just $\left(S^{2}\right)^{\times n}$, i.e. $n$ copies of the 2 -sphere obtained by attaching an $n$-torus $T^{n}$ to each point in the interior of the cuboid. Toric varieties are currently of much interest in string theory, where they play an important role in the construction of CalabiYau manifolds, which are necessary for compact extra dimensions (see e.g. [6] and references).

The purpose of this work is to construct other types of solution to the equation (1.1) for the case of constant scalar curvature $\kappa$, that is

$$
\partial_{j} \partial_{k} u^{j k}=-\kappa, \quad \text { constant. }
$$

Henceforth, following [11], (1.7) is referred to as Abreu's equation. The existence of a Kähler metric of constant scalar curvature is intimately related to the notion of stability of complex projective varieties [11], and for toric surfaces Donaldson has shown the connection with the existence of a lower bound for the Mabuchi functional $[26]$

$$
\mathcal{F}(u)=-\int_{P} \log \operatorname{det}\left(u_{j k}\right) d \mu+\int_{\partial P} u d \sigma-\kappa \int_{P} u d \mu .
$$

In the above, $d \mu$ is Lebesgue measure on $\mathbb{R}^{n}, d \sigma$ is a suitable measure on the boundary $\partial P$ of the polytope, and the PDE (1.7) is the Euler-Lagrange equation $\delta \mathcal{F}=0$ for the Mabuchi functional. For $n>1$ we show that Abreu's equation admits solutions of a different form compared with (1.6), namely $O(n)$ invariant similarity solutions, which are presented in section 2 . The ordinary differential equation for these rotation invariant solutions can be derived from a Lagrangian which appears as a natural reduction of the Mabuchi functional.

Rather than being geometric, our motivation for considering the partial differential equation (PDE) (1.7) comes from the theory of integrable systems. Taking derivatives of (1.6) we have

$$
u_{j k}=\frac{1}{2} \sum_{l=1}^{d} \frac{c_{l, j} c_{l, k}}{\ell_{l}(\mathbf{x})},
$$

where the constants $c_{l, j}$ are the coefficients of the affine functions $\ell_{l}(\mathbf{x})=c_{l, 0}+$ $\sum_{j=1}^{n} c_{l, j} x^{j}$. The expression (1.9) is reminiscent of the potential for vanishing rational solutions [25, 34] of the integrable Kadomtsev-Petviashvili (KP) equation,

$$
\frac{\partial}{\partial x}\left(4 \frac{\partial u}{\partial t_{3}}-12 u \frac{\partial u}{\partial x}-\frac{\partial^{3} u}{\partial x^{3}}\right)-3 \frac{\partial^{2} u}{\partial t_{2}^{2}}=0 .
$$

which takes the form

$$
w=-\sum_{l=1}^{d} \frac{1}{x-x_{l}(\underline{\underline{t}})}, \quad u=-2 \frac{\partial w}{\partial x},
$$


where $\underline{t}$ denotes the dependence of the poles on the times $t_{2}, t_{3}$ (or an infinite sequence of such times in the full KP hierarchy). The dynamics of the $d$ poles $x_{l}(\underline{t})$ is governed by the integrable Calogero-Moser system for $d$ particles. The potential $w$ in (1.11) is a central object in the Sato formulation of KP theory [29], determining the dressing operator $W=1+w \partial_{x}^{-1}+\ldots$ for the pseudo-differential Lax operator $L=W \partial_{x} W^{-1}$.

It is also interesting to note that the sixth Painlevé equation has special solutions of the form (1.6) for $n=1=d$, and there is a similar class of solutions of the generalised WDVV equations of topological field theory. ${ }^{1}$ More precisely, it was proved in [36] that the function

$$
F(\mathbf{x})=\sum_{\alpha \in \mathcal{A}_{+}} m_{\alpha}(\alpha, \mathbf{x})^{2} \log (\alpha, \mathbf{x})^{2}
$$

is a prepotential for the generalised WDVV equations. The $m_{\alpha}$ are multiplicities and $\alpha$ are vectors in the (generalised) positive half root system $\mathcal{A}_{+}$, while $($,$) denotes$ inner product. The derivative of the prepotential (1.12) with respect to any of its arguments is of a form very similar to (1.6), modulo rescaling and the addition of an affine function.

The similarity between (1.9) and the rational KP potential (1.11), as well as the possible connection with the WDVV equations, raises the question of whether Abreu's equation (1.7) might be integrable in some sense. The conjecture of Ablowitz-RamaniSegur [2] asserts that (possibly after a change of variables) all reductions of integrable PDEs should possess the Painlevé property that all solutions are single-valued around all movable singularities. To avoid begging the question, the adjective "integrable" applied to a PDE is taken to mean that it has a Lax pair, ensuring solvability by the inverse scattering transform [3], and/or it has infinitely many commuting symmetries [27]. In order to treat Abreu's equation in the light of the Ablowitz-Ramani-Segur conjecture, it is necessary to seek similarity reductions of (1.7) and examine the structure of their singularities in the complex plane. The similarity reduction to rotation invariant solutions in section 2 yields a second order non-autonomous ordinary differential equation (ODE). Having considered the basic local existence results for solutions of the ODE in section 3, the asymptotic analysis around singular points is carried out in section 4 . In section 5 it is proved that solutions that are positive and monotone increasing on the positive real semi-axis must reach a singularity at a finite point $r^{*}$. Such solutions are constructed using a nonlinear integral equation in section 6 ; they correspond to rotation invariant solutions of (1.7) on the interior of a ball of radius $r^{*}$ in $\mathbb{R}^{n}$. Further discussion is reserved for the conclusions in the final section. Before proceeding, we further outline the geometric background to Abreu's equation in the following subsection, where we also demonstrate its invariance under the action of the affine group.

1.1. Geometrical background on toric varieties. Since their definition in the 1970s, toric varieties have become important in algebraic geometry [10]. They have also inspired a great deal of interest among theoretical physicists, since they play an important part in the construction of mirror pairs of Calabi-Yau manifolds [6]. In the algebro-geometric setting, a toric variety is naturally defined as an irreducible variety $V$ such that the complex torus $\left(\mathbb{C}^{*}\right)^{n}$ is a Zariski open subset of $V$, and the action of the complex torus on itself extends to an action on $V$ (see [10] and references for other definitions). Taking complex coordinates on $\left(\mathbb{C}^{*}\right)^{n}$ given by $\left(w_{1}, \ldots w_{n}\right)$, so that the

\footnotetext{
${ }^{1}$ I am grateful to Tasos Tongas and Eugene Ferapontov for these interesting observations.
} 
action of the torus on itself is componentwise, we extend these to coordinates on $V$, and then it is convenient to change variables to

$$
z_{j}=\log w_{j}=\zeta_{j}+i \theta_{j} .
$$

The action of the real torus $T^{n}=\left\{\left(e^{i \psi_{1}}, \ldots, e^{i \psi_{n}}\right)\right\}$ on the real and imaginary parts gives

$$
\zeta_{j} \rightarrow \zeta_{j}, \quad \theta_{j} \rightarrow \theta_{j}+\psi_{j}
$$

A toric variety, which is an $n$-dimensional complex manifold, can further be given a symplectic structure compatible with its complex structure, or in other words it has a Kähler structure. The Kähler two-form is

$$
\omega=\frac{i}{2} \phi^{j k} d z_{j} \wedge d \bar{z}_{k},
$$

where $\phi^{j k}$ denotes the Hessian of the Kähler potential $\phi=\phi\left(\zeta_{1}, \ldots, \zeta_{n}\right)$, so that

$$
\phi^{j k}=\frac{\partial^{2} \phi}{\partial \zeta_{j} \partial \zeta_{k}} .
$$

Since $\phi$ is independent of the angle variables $\theta_{1}, \ldots, \theta_{n}$, the symplectic form $\omega$ is invariant under the action of the real torus $T^{n}$. In order to see how Abreu's equation (1.7) arises, we need to change to canonical symplectic coordinates on the toric variety $V$.

In the symplectic picture, there is a Hamiltonian action of $T^{n}$ on $V$, considered as a symplectic manifold of real dimension $2 n$, with moment map

$$
m: V \longrightarrow \mathbb{R}^{n} \text {. }
$$

It turns out that the image of the moment map is $m(V)=P$, a convex polytope in $\mathbb{R}^{n}$ (see [14]). Moreover, the components of the moment map

$$
m^{j}\left(\zeta_{1}, \ldots, \zeta_{n}\right)=: x^{j}=\frac{\partial \phi}{\partial \zeta_{j}}
$$

define a Legendre transformation, with $\left\{x_{j}, \theta_{j}\right\}_{j=1}^{n}$ being canonical symplectic coordinates on $V$. The symplectic potential $u$, defined on the polytope $P$, is then given by

$$
u\left(x^{1}, \ldots, x^{n}\right)=x^{j} \zeta_{j}-\phi\left(\zeta_{1}, \ldots, \zeta_{n}\right) .
$$

In the canonical coordinates, the Kähler form is

$$
\omega=d x^{j} \wedge d \theta_{j},
$$

and the natural metric on $V$ is given by (1.4). Moreover, Abreu has shown that the curvature $S=S\left(x^{1}, \ldots, x^{n}\right)$ of such a metric is given in terms of the derivatives of the symplectic potential by the formula $(1.1)$ (see $[4,5]$ ). In order to determine which projective toric varieties admit a Kähler metric of constant scalar curvature, the solution of the PDE (1.7) must be considered on the interior of a polytope $P$. 
As far as we are aware [12], the only exact solutions known at present correspond to direct products of copies of complex projective space,

$$
V=\mathbb{C P}^{n_{1}} \times \mathbb{C P}^{n_{2}} \times \ldots \times \mathbb{C P}^{n_{m}} .
$$

In this case for the $j$ th component $\mathbb{C P}^{n_{j}}$ the corresponding polytope is just a simplex in real $n_{j}$-dimensional space, and the solutions of (1.7) all have the form (1.6), with the affine functions $\ell_{l}(\mathbf{x})$ defining the facets of the polytope.

The aim of what follows is not to seek solutions of Abreu's equation (1.7) defined on polytopes $P$, but rather to study the properties of another class of solutions, namely rotation invariant solutions. Because Abreu's equation (1.7) is an autonomous partial differential equation, an arbitrary shift $\mathbf{x} \rightarrow \mathbf{x}+\delta$ in the independent variables maps solutions to solutions. Furthermore, because of the special structure of the equation, it is natural to consider the action of the group $\operatorname{GL}(n, \mathbb{R})$ on the coordinate vector $\mathbf{x}$. These two group actions combine to give an action of the affine group, which is the semi-direct product $G=\mathrm{GL}(n, \mathbb{R}) \ltimes \mathbb{R}^{n}$.

Proposition 1.1. Abreu's equation (1.7) is invariant under the action of the group $G=\mathrm{GL}(n, \mathbb{R}) \ltimes \mathbb{R}^{n}$, such that $\mathbf{x} \rightarrow \tilde{\mathbf{x}}=\mathbf{K} \mathbf{x}+\delta$.

Proof. Clearly the action of $\mathbb{R}^{n}$ by shifts in the coordinates has no effect, because the PDE (1.7) is autonomous. Denoting an element of $\mathrm{GL}(n, \mathbb{R})$ by $\mathbf{K}=\left(K_{k}^{j}\right)$, we have

$$
\tilde{x}^{j}=K_{k}^{j} x^{k}+\delta^{j} .
$$

It is also convenient to denote the inverse of $\mathbf{K}$ by $\mathbf{L}$, so that

$$
L_{p}^{q} K_{q}^{r}=\delta_{p}^{r}=K_{p}^{q} L_{q}^{r} .
$$

under the action of the group $G$ (with the Einstein summation convention being assumed as before). Now suppose that $u(\mathbf{x})$ is a solution of (1.7), and define

$$
v(\mathbf{x})=u(\tilde{\mathbf{x}}) .
$$

Then in terms of the transformed variables, we have that

$$
\tilde{\partial}_{j} \tilde{\partial}_{k} \tilde{u}^{j k}=-\kappa .
$$

To show invariance of the PDE, we must show that $v$ is also a solution of (1.7).

The derivatives transform in the obvious way as

$$
\partial_{l}=K_{l}^{j} \tilde{\partial}_{j}
$$

so that we have

$$
\partial_{j} \partial_{k} v^{j k}=K_{j}^{l} K_{k}^{m} \tilde{\partial}_{l} \tilde{\partial}_{m} v^{j k} .
$$

But now the inverse Hessian of $v$ satisfies

$$
v^{j k}\left(\partial_{k} \partial_{l} v\right)=\delta_{l}^{j}=v^{j k} K_{k}^{m} K_{l}^{n} \tilde{u}_{m n},
$$

where $\tilde{u}_{m n}$ denotes the components of the Hessian of $u(\tilde{\mathbf{x}})$, whence

$$
v^{j k}=L_{p}^{j} \tilde{u}^{p q} L_{q}^{k} .
$$


Substituting the latter expression into (1.13) gives

$$
\partial_{j} \partial_{k} v^{j k}=K_{j}^{l} K_{k}^{m} \tilde{\partial}_{l} \tilde{\partial}_{m} L_{p}^{j} L_{q}^{k} \tilde{u}^{p q}=\delta_{p}^{l} \delta_{q}^{m} \tilde{\partial}_{l} \tilde{\partial}_{m} \tilde{u}^{p q}=-\kappa
$$

as required. $\square$

Fixing a choice of scale and origin naturally leads us to consider rotationally invariant solutions in the next section.

2. Similarity solutions. For each $n$, Abreu's equation (1.7) has rotation invariant solutions, with the potential $g$ being a function of the radial distance only, that is

$$
u=u(r), \quad r=|\mathbf{x}| .
$$

In that case the Hessian matrix and its inverse are given by

$$
\mathbf{H}=\frac{f}{r} \mathbf{1}+\frac{1}{r}\left(\frac{f}{r}\right)^{\prime} \mathbf{x x}^{T}, \quad \mathbf{H}^{-1}=\frac{r}{f} \mathbf{1}+\left(\frac{1}{r^{2} f^{\prime}}-\frac{1}{r f}\right) \mathbf{x x}^{T},
$$

where

$$
f=u^{\prime}
$$

and the prime denotes $d / d r$. Since the Hessian $\mathbf{H}$ is given entirely in terms of the function $f$ and its derivative, and the potential $u$ is obtained from $f$ by a quadrature, in what follows we need only consider the equation for $f$. We may formulate this precisely as

Proposition 2.1. For any dimension n, Abreu's equation (1.7) has rotation invariant similarity solutions. The potential u is given by a quadrature,

$$
u(\mathbf{x})=\int^{r} f(s) d s, \quad r=|\mathbf{x}|,
$$

where $f(r)$ is a solution of the second order differential equation

$$
f^{\prime \prime}=\left(\frac{\kappa}{n} r-\frac{\lambda}{r^{n-1}}-\frac{(n-1)}{f}\right)\left(f^{\prime}\right)^{2}+\frac{(n-1)}{r} f^{\prime},
$$

for arbitrary constant $\lambda$.

Proof. Substituting the form (2.1) of the inverse Hessian matrix into (1.7) yields the following third order ordinary differential equation (ODE) for $f$ :

$$
\left(n+r \frac{d}{d r}\right)\left(\frac{A^{\prime}}{r}+r B^{\prime}+(n+1) B\right)=-\kappa .
$$

In the above, $A$ and $B$ denote the quantities

$$
A=\frac{r}{f}, \quad B=\frac{1}{r^{2} f^{\prime}}-\frac{1}{r f} .
$$

After an integration, the third order ODE yields

$$
\frac{A^{\prime}}{r}+r B^{\prime}+(n+1) B=\lambda r^{-n}-\frac{\kappa}{n},
$$


for constant $\lambda$. Upon substituting for $A, B$ from (2.3), the equation (2.4) is a second order ODE for $f=f(r)$, which takes the explicit form (2.2).

For the purposes of analysis, it is sometimes convenient to rewrite (2.2) in the form

$$
\frac{d}{d r} \log \left(\frac{f^{n-1} f^{\prime}}{r^{n-1}}\right)=\left(\frac{\kappa}{n} r-\lambda r^{1-n}\right) f^{\prime} .
$$

In the context of compact manifolds, $\kappa$ is a positive constant, and for any (non-zero) $\kappa$ it is straightforward to rescale $f \rightarrow f / \kappa$ and set $\kappa=1$, but we choose to leave $\kappa$ arbitrary. We now consider some explicit solutions.

Trivial solution: Clearly for any $n$ the ODE (2.2) has the trivial solution

$$
f=\text { constant. }
$$

In this case the inverse Hessian $\mathbf{H}^{-1}$ in (2.1) becomes infinite, and hence this does not correspond to a bona fide solution of Abreu's equation (1.7).

General solution for $n=1$ : In the case $n=1$, Abreu's equation is itself an ODE equivalent to (2.2), which is easily integrated to obtain the general solution

$$
f=\frac{1}{\rho} \log \left(\frac{\rho-\lambda+\kappa r}{\rho+\lambda-\kappa r}\right)+\alpha,
$$

so that the potential is

$$
u=\rho^{-1}\left(r-r_{-}\right) \log \left(r-r_{-}\right)+\rho^{-1}\left(r_{+}-r\right) \log \left(r_{+}-r\right)+\alpha r+\beta, \quad r_{ \pm}=(\lambda \pm \rho) / \kappa,
$$

for arbitrary constants $\rho>0, \alpha, \beta$. Up to shifting by an affine function, the solution for $n=1$ is of the form (1.6), with $u(r)$ defined on a single interval $\left(r_{-}, r_{+}\right)$in $\mathbb{R}$, which by the construction of [14] leads to a toric metric on the sphere $S^{2}$, or equivalently $\mathbb{C P}^{1}$.

Special solution and symmetry reduction for $\lambda=0$ : When $\lambda=0$ (and with $\kappa \neq 0$ ), there there is an exact solution that is singular at the origin, namely

$$
f=\frac{2 n^{2}}{\kappa} r^{-1}
$$

Furthermore, (2.2) is invariant under the one-parameter group of scaling symmetries $r \rightarrow \mu r, f \rightarrow \mu^{-1} f$, so by standard methods of symmetry reduction [30] it is possible to reduce the order. Introducing the new scale-invariant independent variable $y=r f$, and the dependent variables $v=v(y)=-\log r, w=d v / d y$, it reduces to a first order equation for $w(y)$ :

$$
\frac{d w}{d y}=y(2 n-\kappa y) w^{3}+(3 n-2 \kappa y) w^{2}+((n-1) / y-\kappa) w .
$$

(This was presented as an example in [20].)

Special solution for $\kappa=0$ : When $\kappa=0$ and $\lambda=0$ the equation (2.2) can be integrated exactly to give

$$
f=\left(C+D r^{n}\right)^{1 / n},
$$


with two arbitrary constants $C, D$. Although zero curvature does not arise in the context of compact toric varieties, it turns out that the special case $n=2$ and $\kappa=0$ is of geometric interest for so called "self-dual" metrics that fit into the twistor framework [12]. The special case $\kappa=0$ also admits a scaling invariance under symmetry $r \rightarrow \mu r$, $f \rightarrow \mu^{n-1} f$, so as for the case $\lambda=0$ it is possible to make a symmetry reduction to a first order equation, which we omit here.

For $n>1$ in general we are unable to integrate (2.2) explicitly, and must resort to local and asymptotic analysis. Before embarking on this in the following sections, we consider what sort of initial value problem for the ODE is relevant in the geometric context. Since $r$ is the radial coordinate in $\mathbb{R}^{n}$, ideally we would like solutions defined on some interval $\left[0, r^{*}\right.$ ) of the positive real semi-axis (possibly with $r^{*}=\infty$ ). Starting from initial data specified away from the origin at $r=\eta>0$, the ODE (2.2) is regular. However, because $r=0$ is a singular point, it is not possible to take initial data at the origin - instead we would need to specify some asymptotic behaviour, depending on whether this is a regular or irregular singularity [37,35]. Clearly the right hand side of (2.2) is also singular at $f=0$. It is easy to see that given $f^{\prime}(\eta) \neq 0$, the solution (as long as it exists) cannot have stationary points for $r>\eta$, since the right hand side of (2.2) vanishes when $f^{\prime}=0$; thus, by the uniqueness of the solution to the initial value problem (see next section), integrating the ODE from any point $\zeta$ where the gradient vanishes gives only the constant solution $f(r)=f(\zeta)=$ constant. The following result is immediate:

Lemma 2.2. Suppose that initial data is specified for the ODE (2.2) at a point $r=\eta>0$, with $f(\eta), f^{\prime}(\eta)$ both nonzero. Then the solution $f(r)$ for $r>\eta$ remains positive (negative) for $f(\eta)>0(f(\eta)<0)$, and for $f^{\prime}(\eta)>0$ or $f^{\prime}(\eta)<0$ it is monotone (increasing or decreasing, respectively), as long as it exists.

For a solution of the ODE on the interval $\left[0, r^{*}\right)$, the components of the Hessian matrix $\mathbf{H}$ determine a metric (1.2) on the ball of radius $r^{*}$. The eigenvalues of $\mathbf{H}$ in (2.1) are $f / r$ (repeated $n-1$ times) and $f^{\prime}$. Thus if we require a Riemannian metric given by a positive definite Hessian, then we should consider only solutions of (2.2) defined with both initial data positive, i.e. $f(\eta)>0, f^{\prime}(\eta)>0$. The signs of $f$ and $f^{\prime}$ remain constant and the Lemma also holds when the solution is analytically continued to $r<\eta$, provided it exists. However, a priori we have no guarantee that when the solution is continued back towards $r=0$ (solving the initial value problem in reverse) it will not reach a singularity at some point $r=\eta^{\prime}$ with $0<\eta^{\prime}<\eta$. In the next section the local existence results in the complex plane are presented, while the asymptotic analysis of section 4 shows that both algebraic and logarithmic branch points are possible. The results in section 5 imply that the real solutions with both initial data positive must hit a singularity at a finite point on the positive real semi-axis.

Before analysing the ODE further, it is interesting to note that it admits a Lagrangian formulation.

Proposition 2.3. The ODE (2.2) can be derived as the stationarity condition $\delta S=0$ for the action

$$
S=\int_{r_{1}}^{r_{2}} L\left(f, f^{\prime}, r\right) d r
$$


with the Lagrangian

$$
L=r^{n-1} \log \left(f^{n-1} f^{\prime}\right)+\left(\lambda-\frac{\kappa}{n} r^{n}\right) f .
$$

Proof. This follows from a straightforward calculation of the Euler-Lagrange equation

$$
\frac{\partial L}{\partial f}-\frac{d}{d r}\left(\frac{\partial L}{\partial f^{\prime}}\right)=0 .
$$

The Lagrangian (2.9) is non-autonomous, since it depends explicitly on the independent variable $r$ (corresponding to time in the physical interpretation of Lagrangian mechanics). In fact the action $S$ in (2.8) must have its origin in the Mabuchi functional $\mathcal{F}$ for the PDE (1.7). Observe that for the reduction to rotation invariant solutions, the nonlinear part of (1.8) becomes

$$
\int \log \operatorname{det}\left(u_{j k}\right) d \mu=\int \log \operatorname{det} \mathbf{H} d \mu=\int \log \left(\frac{f^{n-1} f^{\prime}}{r^{n-1}}\right) r^{n-1} d r d \Omega,
$$

where $d \Omega$, the angular part of the measure, is the volume element on the sphere $S^{n-1}$. For $O(n)$ invariant solutions the angular part decouples and gives an overall prefactor, and then after removing a term depending only on $r$ the final expression in (2.10) is precisely equivalent to the logarithmic term in the action (2.8). The derivation of the $\lambda$ and $\kappa$ terms in the Lagrangian (2.9) is not quite so obvious from the linear part of the Mabuchi functional (1.8), since it requires careful evaluation of the surface integral (converted to a volume integral with Stokes' theorem), but the removal of dependence on $u$ could be achieved by introducing $\lambda$ as a Lagrange multiplier for the constraint $u^{\prime}=f$.

3. Local existence. The right hand side of equation (2.2) becomes singular at $r=0$ and $f=0$. By standard existence theorems [21], if regular initial data $f(\eta)=f_{\eta} \neq 0, f^{\prime}(\eta)=p_{\eta}$ is specified at any point $0 \neq \eta \in \mathbb{C}$, then (2.2) has a unique analytic solution in the neighbourhood of $r=\eta$. For the purposes of applications to (positive definite) metric tensors, the important question is whether there are real solutions around a point $r=\eta>0$ with $f_{\eta}>0, p_{\eta}>0$, and with an analytic continuation along the real axis. For completeness, below a lower bound is presented on the radius of the domain of analyticity around a real point for the case of positive initial data $f_{\eta}, p_{\eta}$; such bounds are easily modified in the general case.

It is convenient to rewrite (2.2) as the first order system

$$
f^{\prime}=p, \quad p^{\prime}=\left(\frac{\kappa r}{n}-\frac{\lambda}{r^{n-1}}-\frac{(n-1)}{f}\right) p^{2}+\frac{(n-1)}{r} p .
$$

In that case given the real initial data $f_{\eta}>0, p_{\eta}>0$ at $r=\eta>0$, then for any $a$ and $b$ with $0<a<\eta$ and $0<b<f_{\eta}$, it is clear that the right hand sides of the system (3.1) are analytic functions of $r, f, p$ in the domain $|r-\eta| \leq a,\left|f-f_{\eta}\right| \leq b$, $\left|p-p_{\eta}\right| \leq b$. In this domain, the right hand sides of the system admit the upper bounds

$$
|p| \leq M_{1}, \quad\left|\left(\frac{\kappa r}{n}-\frac{\lambda}{r^{n-1}}-\frac{(n-1)}{f}\right) p^{2}+\frac{(n-1)}{r} p\right| \leq M_{2},
$$


where

$$
M_{1}:=b+p_{\eta}, \quad M_{2}:=\left(\frac{\kappa}{n}(a+\eta)+\frac{|\lambda|}{(\eta-a)^{n-1}}+\frac{(n-1)}{\left(f_{\eta}-b\right)}\right) M_{1}^{2}+\frac{(n-1)}{(\eta-a)} M_{1} .
$$

Proposition 3.1. The unique analytic solution of the ODE (2.2) satisfying $f(\eta)=f_{\eta}>b>0, f^{\prime}(\eta)=p_{\eta}>0$ must exist in a circle of minimum radius

$$
\rho=a\left(1-e^{-\frac{b}{3 M a}}\right),
$$

around $r=\eta$, where the upper bound $M$ is given in terms of the bounds (3.2) by

$$
M=\max \left\{M_{1}, M_{2}\right\} .
$$

Proof. This follows by a direct application of the result of section 12.21 in [21].

Starting from points within this circle, the solution may be analytically continued in any direction in the complex plane, until it reaches a singularity. In the next section we see that both movable algebraic and logarithmic branch points are possible, and the fixed singular points of the ODE are also analysed.

4. Asymptotic analysis. In order to analyse the different possible singular behaviours of the solutions of the ODE (2.2), it is necessary to find all the dominant balances [23] and thence obtain asymptotic expressions in the neighbourhood of singular points. Linear differential equations can have only fixed singularities at the points in the complex plane where the coefficient functions are singular. However, in the case of nonlinear equations such as (2.2) there can also be movable singularities, whose position is not fixed but depends on the initial conditions. In this section formal asymptotic expansions around both fixed and movable singularities are presented for solutions of equation $(2.2)$. The reader is referred to $[16,21]$ for more detailed discussions of singularity analysis.

4.1. Expansions at $r=0$. There are several different types of asymptotic behaviour near the origin.

Proposition 4.1. For $n \geq 3$ and $\lambda \neq 0$ there are three types of asymptotic behaviour of the solutions of (2.2) as $r \rightarrow 0$ :

(a) $f \rightarrow f_{0} \neq 0$, with the leading order behaviour of the derivative given by

$$
f^{\prime \prime} \sim \frac{(n-1)}{r} f^{\prime}
$$

(b) $f \rightarrow f_{0} \neq 0$, with the leading order behaviour of the derivative given by

$$
f^{\prime \prime} \sim-\frac{\lambda}{r^{n-1}}\left(f^{\prime}\right)^{2}+\frac{(n-1)}{r} f^{\prime}
$$

(c) $f \rightarrow 0$, with

$$
f \sim-\frac{n(n-2)}{\lambda(n-1)} r^{n-1} .
$$


Proof. To determine the possible asymptotic behaviours at the origin, it is necessary to determine which combinations of terms in the differential equation (2.2) can balance at leading order. Clearly the $\kappa r / n$ term is always of lower order than the $\lambda / r^{n-1}$ term in front of $\left(f^{\prime}\right)^{2}$, and hence does not enter into the leading order analysis. By Lemma 2.2 we know that $f$ is a monotone function. First we consider the case that $r^{n-1} / f=o(1)$ as $r \rightarrow 0$, so that only the three terms $f^{\prime \prime}, \lambda\left(f^{\prime}\right)^{2} / r^{n-1}$ and $(n-1) f^{\prime} / r$ can potentially balance each other at leading order. It turns out that only two possible balances of this kind are consistent. In case (a) given by 4.1, two terms balance and upon integration we have $f^{\prime} \sim c n r^{n-1}$ for some constant $c$, whence $f \sim f_{0}+c r^{n}$ with $f_{0} \neq 0$ to ensure $r^{n-1} / f=o(1)$. There are no other consistent balances with just two of these three terms balancing, so the remaining possibility is that all three terms are of the same order, which yields case (b) above. Integrating the equation (4.2) leads to $f^{\prime} \sim r^{n-2} / \lambda$ and hence $f \sim f_{0}+r^{n-1} /(\lambda(n-1))$ with $f_{0} \neq 0$ for $r^{n-1} / f=o(1)$.

Next we consider $f / r^{n-1}=o(1)$. The $f^{\prime \prime}$ and $(n-1) f^{\prime} / r$ terms are always of the same magnitude, but in order to avoid reducing to case (a) (which would contradict $\left.f / r^{n-1}=o(1)\right)$ we are forced to assume that these terms balance with $(n-1)\left(f^{\prime}\right)^{2} / f$. This gives

$$
f^{\prime \prime}+\frac{(n-1)}{f}\left(f^{\prime}\right)^{2}-\frac{(n-1)}{r} f^{\prime}=f^{\prime} \frac{d}{d r} \log \left(\frac{f^{\prime} f^{n-1}}{r^{n-1}}\right) \sim 0,
$$

and integrating gives leading order behaviour as in (2.7), which again contradicts $f / r^{n-1}=o(1)$. The last case we must consider is $f=O\left(r^{n-1}\right)$ as $r \rightarrow 0$, which immediately yields case (c) as in (4.3).

We can develop these leading order asymptotics into formal series around $r=0$, as follows:

- For any $\lambda,(2.2)$ admits the formal expansion

$$
f \sim f_{0}+\sum_{j=0}^{\infty} a_{j} r^{n+j}, \quad r \rightarrow 0,
$$

where $f_{0} \neq 0$ and $a_{0} \neq 0$ are arbitrary constants, and the other coefficients are determined recursively by

$j(n+j) a_{j}=-\lambda \sum_{k=0}^{j-1}(n+k)(n+j-k-1) a_{k} a_{j-k-1}+G_{j}\left(f_{0}, a_{0}, \ldots, a_{j-2}\right), j \geq 1$,

for certain functions $G_{j}$. These functions $G_{j}$ are difficult to write in a compact form, since while the first term on right hand side of the recurrence is of degree two in $a_{k}$, the degree of $G_{j}$ increases with $j$; nevertheless they are easily obtained recursively by substitution in the differential equation (2.2). For solutions with this asymptotic behaviour, $\operatorname{det} \mathbf{H}(r) \rightarrow n a_{0} f_{0}^{n-1}$ as $r \rightarrow 0$.

- For $\lambda \neq 0$ there is an alternative expansion

$$
f \sim f_{0}+\frac{1}{\lambda(n-1)} r^{n-1}+\sum_{j=0}^{\infty} b_{j} r^{n+j}, \quad r \rightarrow 0,
$$

which depends on just one arbitrary parameter $f_{0} \neq 0$, with the coefficients determined by a recursion

$$
(j+2)(j+n) b_{j}=H_{j}\left(f_{0}, b_{0}, \ldots, b_{j-1}\right)
$$


with a sequence of functions $H_{j}$, whose exact form is omitted. For $n=2$, $b_{0}=-1 /\left(4 \lambda^{2} f_{0}\right)$, while $b_{0}=0$ for $n \geq 3$. In this case $\operatorname{det} \mathbf{H}(r) \rightarrow \pm \infty$ as $r \rightarrow 0$.

- For $\lambda \neq 0$ and $n \neq 2$ there is another formal expansion in (2.2) that contains no arbitrary parameters, namely

$$
f \sim-\frac{n(n-2)}{\lambda(n-1)} r^{n-1}+\sum_{j=0}^{\infty} c_{j} r^{n+j}
$$

for $n \neq 2$. The coefficients satisfy a recurrence of the form

$$
\left((j+2)(j+n)-(n-1)^{3}\right) c_{j}=J_{j}\left(c_{0}, \ldots, c_{j-1}\right),
$$

for certain functions $J_{j}$; it turns out that $c_{0}=0$. With these asymptotics at the origin, $\operatorname{det} \mathbf{H}(r) \rightarrow 0$ as $r \rightarrow 0$. However, while the leading part of the asymptotic series (4.6) is certainly correct, the complete expansion may break down at a resonance, i.e. if the quadratic in $j$ on the left hand side of (4.7) vanishes. This can only occur for some value of $j$ and for particular $n$ such that the discriminant $4 n^{3}-11 n^{2}+8 n$ is a perfect square; this is equivalent to the existence of integer points $(x, y)$ on the elliptic curve $y^{2}=4 x^{3}-11 x^{2}+8 x$. It turns out that $(0,0),(1, \pm 1)$ and $(2, \pm 2)$ are the only integer points on this curve ${ }^{2}$ but none of these values are relevant here since the leading order in (4.6) requires $x=n \geq 3$; hence there are no resonances and the expansion (4.6) is consistent.

- In the case $\lambda=0$, there is also the exact solution (2.6) that is singular at the origin.

Remark. We are grateful to one of the referees for pointing out that, with reference to the type (a) asymptotic behaviour, setting $f=f_{0}+r^{n} \psi$ leads to a regular singularity for $\psi(r)$ at $r=0$. In that case we can make use of some standard results on asymptotic series $[37,35]$. However, in section 6 we prefer to present a direct approach to these solutions, using an integral equation.

4.2. The point at infinity. Although it will become clear that this will be irrelevant for the construction of a solution of (1.7) in a neighbourhood of the origin, here we present the two types of asymptotic expansion at infinity for the equation $(2.2)$ :

- In one case, $f$ tends to zero:

$$
f \sim \frac{2 n^{2}}{\kappa} r^{-1}+\sum_{j=0}^{\infty} d_{j} r^{-j-2}, \quad r \rightarrow \infty,
$$

with the coefficients $d_{j}$ determined uniquely by a recurrence

$$
\left(j^{2}-(n-2) j-3 n+1\right) d_{j}=K_{j}\left(d_{0}, \ldots, d_{j-1}\right) .
$$

The discriminant of the quadratic $j^{2}-(n-2) j-3 n+1$ is $n^{2}+8 n$, and $n=1$ is the only positive integer for which this can be a perfect square, so there are no resonances.

\footnotetext{
${ }^{2}$ Thanks to John Merriman for checking this.
} 
- In the other case, $f$ is asymptotic to a nonzero constant, i.e.

$$
f=\ell_{0}+\ell_{1} r^{-1}+o\left(r^{-1}\right), \quad r \rightarrow \infty,
$$

with

$$
\ell_{0} \neq 0 \quad \text { (arbitrary), } \quad \ell_{1}=\frac{n(n+1)}{\kappa} .
$$

However, if this is to be extended to a complete asymptotic expansion

$$
f \sim \sum_{j=0}^{\infty} \ell_{j} r^{-j}
$$

then the recurrence for the coefficients takes the form

$$
j(j-n-2) \ell_{j}=L_{j}\left(\ell_{0}, \ldots, \ell_{j-1}\right), \quad L_{0} \equiv 0,
$$

which clearly has a resonance at $j=n+2$ (as well as the resonance $j=0$ corresponding to the fact that $\ell_{0}$ is arbitrary). Thus the simple power series expansion is inconsistent unless $L_{n+2}$ is identically zero. For example, when $n=2$ we find at order $r^{-4}$ that

$$
L_{4}=-\frac{3 \ell_{1}^{4}}{4 \ell_{0}^{3}} \neq 0, \quad \text { since } \quad \ell_{1}=\frac{6}{\kappa}
$$

so the inconsistency can only be rectified by including a term proportional to $r^{-4} \log r$ in the asymptotics, which in turn requires infinitely many terms of the form $r^{-j}(\log r)^{k}$ to ensure a consistent asymptotic expansion.

There are some standard theorems concerning asymptotic expansions of ODEs around irregular singular points at infinity [37], and these have recently been extended to more general classes of equations [35]. To consider (2.2) in the light of such results, it should be rewritten as a system

$$
\frac{1}{r} \frac{d}{d r}\left(\begin{array}{c}
f \\
q
\end{array}\right)=\left(\begin{array}{c}
f q r^{-1} \\
\left(\kappa / n-\lambda r^{-n}\right) f q^{2}-n q^{2} r^{-1}+(n-1) q r^{-2}
\end{array}\right), \quad q:=\frac{d}{d r} \log f .
$$

The vector function on the right hand side is holomorphic for $(r, f, q) \in\left(\mathbb{C}^{*} \cup\{\infty\}\right) \times$ $\mathbb{C}^{2}$, and $r=\infty$ is an irregular singular point of Poincaré rank 2 for the system (4.10). As a function of $(f, q) \in \mathbb{C}^{2}$, the linear part of this vector is degenerate (in fact, zero) at $r=\infty$, which means that Wasow's Theorem 33.1 in [37] does not apply. Nevertheless, recent developments (see [35] and references therein) imply that given the formal expansion (4.8) in powers of $r^{-1}$, there must exist a proper solution of the system (4.10) which has this asymptotic expansion in some sector of angle $\pi / 2$. However, these considerations do not apply to more general asymptotics such as (4.9), since in that case the formal power series must be augmented by logarithmic terms due to resonances. Clearly the same framework can be applied to the singular point at $r=0$ by transforming it to $z=\infty$ for the variable $z=r^{-1}$, but in section 6 below we prefer to make a direct construction of solutions with the asymptotics (4.4) at $r=0$. 
4.3. Other fixed singularities. The ODE (2.2) also admits singular expansions at $n$ special points in the complex plane. The function

$$
Q(r)=\frac{\kappa}{n} r-\frac{\lambda}{r^{n-1}}
$$

appearing on the right hand side of (2.2) vanishes at the points $r=\rho_{j}$, where

$$
\rho_{j}=e^{2 \pi i j / n}\left(\frac{\lambda n}{\kappa}\right)^{1 / n}, \quad j=0, \ldots, n-1 .
$$

In the neighbourhood of these points $f$ can have the singular behaviour

$$
f=\frac{(n+1)}{Q^{\prime}\left(\rho_{j}\right)}\left(r-\rho_{j}\right)^{-1}+\left(\frac{(n+1)}{\rho_{j} Q^{\prime}\left(\rho_{j}\right)}-\frac{Q^{\prime \prime}\left(\rho_{j}\right)}{2(n-1)}\right)+o(1), \quad r \rightarrow \rho_{j} .
$$

This can be extended to a full formal expansion

$$
f \sim E(r) \equiv \frac{(n+1)}{Q^{\prime}\left(\rho_{j}\right)}\left(r-\rho_{j}\right)^{-1}+\sum_{j=0}^{\infty} e_{j}\left(r-\rho_{j}\right)^{j},
$$

where the recurrence to determine the higher terms takes the general form

$$
\left(j^{2}+3 j-n+1\right) e_{j}=E_{j}\left(e_{0}, e_{1}, \ldots, e_{j-1}\right),
$$

for suitable functions $E_{j}$. The recurrence determines all of the coefficients $e_{j}$ uniquely unless it happens that $j^{2}+3 j-n+1=0$ for some $j$. This can only arise for particular values of $n$, namely $n=k^{2}+k-1$, for $k=2,3, \ldots$, in which case the coefficient $e_{k-1}$ is arbitrary, subject to the resonance condition $E_{k-1}=0$. In general the resonance condition will not be satisfied, so the power series expansion $E(r)$ must be augmented with terms in $\log \left(r-\rho_{j}\right)$.

4.4. Movable singularities. The ODE (2.2) admits movable singularities at arbitrary points $r_{0} \neq 0, r_{0} \neq \rho_{j}$ in the complex $r$ plane; the position of such singularities is dependent on the initial data. The movable singularities can take the following forms:

- Algebraic branching: The asymptotic behaviour in the neighbourhood of an algebraic branch point is

$$
f \sim \sum_{j=1}^{\infty} k_{j}\left(r-r_{0}\right)^{j / n}, \quad r \rightarrow r_{0},
$$

where both $r_{0}$ and the constant $k_{1}$ are arbitrary. The coefficients are determined uniquely by a recurrence of the form

$$
\frac{(j-1)(j+n-1)}{n^{2}} k_{j}=\mathcal{G}_{j}\left(k_{1}, \ldots, k_{j-1}\right), \quad \mathcal{G}_{1} \equiv 0 ;
$$

$j=1$ is the only resonance, corresponding to the arbitrariness of $k_{1}$.

- Logarithmic branching: The leading order behaviour in the neighbourhood of a logarithmic branch point is

$$
f=\log \left(r-r_{0}\right)\left(-\frac{1}{Q\left(r_{0}\right)}+o(1)\right), \quad r \rightarrow r_{0},
$$

with the function $Q$ as in (4.11). 
Due to the movable algebraic branching (4.14), the ODE (2.2) is outside the Painlevé class of second order equations whose general solution has no movable singularities other than poles, as described in chapter 14 of [21]. If it has only algebraic branching around movable singularities, an ODE can possess the weak Painlevé property as defined in [32], and there are many examples of integrable ordinary and partial differential equations which have this property (see for instance $[1,9,18]$ ). However, the ODE (2.2) also admits movable logarithmic branch points in its solutions. Logarithmic branching such as (4.15) is taken as a strong indicator of non-integrability in differential equations [2]. A further question is whether the ODE can have movable essential singularities.

5. Connection problems. In order that the solution of the ODE (2.2) should determine a Riemannian metric (1.2) in some spherically symmetric neighbourhood of the origin in $\mathbb{R}^{n}$, it is necessary to consider positive initial data at a point $r=\eta>0$ on the positive real semi-axis as in section 3. This solution is then required to have an analytic continuation for $r<\eta$ back to the fixed singular point $r=0$, but upon continuing the solution to $r>\eta$ it must reach a singularity, as we now demonstrate.

Proposition 5.1. Suppose that positive initial data $f(\eta)=f_{\eta}>0, f^{\prime}(\eta)=p_{\eta}>$ 0 is specified for the ODE (2.2) at any point $r=\eta>0$ on the positive real semi-axis. Then the analytic continuation of the solution reaches a singularity at some finite point $r=r^{*}>\eta$.

Proof. Suppose that the solution exists for all $r \geq \eta$. By Lemma $2.2 f^{\prime}$ is positive and $f$ is monotonically increasing, so that $f(r)>f_{\eta}$ for $r>\eta$ and

$$
f^{\prime \prime}=f^{\prime}\left((Q(r)-(n-1) / f) f^{\prime}+(n-1) / r\right)>\left(Q(r)-(n-1) / f_{\eta}\right)\left(f^{\prime}\right)^{2},
$$

with $Q(r)$ given by (4.11). It is clear that for any $Q^{*}>0$ we have

$$
Q(r)>Q^{*}+(n-1) / f_{\eta}, \quad r>\hat{r} \geq \eta,
$$

for sufficiently large $\hat{r}$. Hence for $r>\hat{r}$ the second derivative is bounded by

$$
f^{\prime \prime}>Q^{*}\left(f^{\prime}\right)^{2}
$$

and this inequality may be integrated to yield

$$
-\frac{1}{f^{\prime}(r)}-Q^{*} r>-\frac{1}{f^{\prime}(\hat{r})}-Q^{*} \hat{r}
$$

But then rearranging gives

$$
\frac{1}{f^{\prime}(r)}<Q^{*}(\hat{r}-r)+\frac{1}{f^{\prime}(\hat{r})} \rightarrow-\infty \quad \text { as } \quad r \rightarrow \infty
$$

which is a contradiction since $f^{\prime}$ must be positive.

Since the positive, monotone increasing solutions have a singularity with $f(r) \rightarrow$ $\infty$ as $r \rightarrow r^{*}$ for some finite $r^{*}$, the analysis of section 4 can be used to determine the asymptotic behaviour there. However, it is well known [21] that second order nonlinear equations may have movable essential singularities which cannot be detected by local asymptotic analysis. Subject to this caveat, we can state the following

Proposition 5.2. Suppose that positive initial data is specified for the ODE (2.2) at $r=\eta>0$ as in Proposition 5.1, and that the solution reaches a singularity 
at $r=r^{*}>\eta$. Then assuming that the ODE does not admit movable essential singularities, the only possible asymptotic behaviour of the solution as $r \rightarrow r^{*}$ is the logarithmic branching (4.15) with $r_{0}=r^{*}$ for $\lambda \leq 0$, and similarly for $\lambda>0$ provided that $r^{*}>\rho_{0}$, the principal value $n$th root in (4.12).

Proof. Having assumed that there are no movable essential singularities, the only possible asymptotic behaviours at singular points are those given in section 4 . For $\lambda \leq 0$ if $f \rightarrow \infty$ as $r \rightarrow r^{*}$ with $r<r^{*}$, then there must be a logarithmic branch (4.15), which for real positive $f$ takes the form

$$
f=\log \left(r^{*}-r\right)\left(-\frac{1}{Q\left(r^{*}\right)}+o(1)\right), \quad r \rightarrow r^{*}, \quad r<r^{*} .
$$

For $\lambda>0$ the same is true except that $r^{*}>\rho_{0}$ is necessary to ensure that $Q\left(r^{*}\right)>0$, so that $f$ has the correct sign in (5.1) as $r \rightarrow r^{*}$. $\square$

A proof of freedom from movable essential singularities for the first Painlevé equation, using the approach originally due to Painlevé, appears in chapter 14 of Ince's book [21], but these arguments have only been made completely rigorous quite recently [17]. Here we shall not attempt to prove the non-existence of movable essential singularities for the ODE (2.2), since this is a very delicate issue. Such essential singularities can be isolated or non-isolated; moreover for equations of third order and above, such as Chazy's equation [8]

$$
w^{\prime \prime \prime}=2 w w^{\prime \prime}-3\left(w^{\prime}\right)^{2},
$$

it is known that there can be natural boundary of analyticity. Bearing this in mind, it is nevertheless straightforward to determine which of the local asymptotic behaviours enumerated in section 4 are allowed as the solution of the initial value problem for (2.2) is analytically continued to $r<\eta$, back towards the origin.

Proposition 5.3. Suppose that positive initial data is given for the ODE (2.2) at $r=\eta>0$ as in Proposition 5.1. Assuming the absence of movable essential singularities, then, if the solution is analytically continued back to $r<\eta$, it can either reach the origin with one of the following behaviours as $r \rightarrow 0$ :

(a) asymptotics (4.4) with $f_{0}>0, a_{0}>0$,

(b) asymptotics (4.5) with $f_{0}>0$, for $\lambda>0$ only,

(c) asymptotics (4.6) for $\lambda<0, n \neq 2$ only; or otherwise:

(d) it has an algebraic branch point (4.14) at some point $r_{0}$, with $0<r_{0}<\eta$ and $k_{1}>0$.

Proof. This is obvious, by a simple enumeration of the various possibilities obtained in section 4 . The constraints on the parameters $f_{0}, a_{0}$ and $k_{1}$ in the various asymptotic expansions are necessary to ensure that $f$ and $f^{\prime}$ are both positive.

Similarly it is possible to consider the different singularities that can occur when the solution of (2.2) is analytically continued along the real axis with one or both of the initial data $f(\eta), f^{\prime}(\eta)$ being negative. This programme was carried out in the preprint [19], although there both the possibility of movable essential singularities and the special fixed singularities (4.12) were neglected. If we restrict to the case where $f$ and $f^{\prime}$ are positive, then given a solution defined on the interval $\left(0, r^{*}\right)$ it is natural to wonder how the position of the movable singularity at $r=r^{*}$ is related to the asymptotics as $r \rightarrow 0$. This can be stated more precisely thus: 
Connection problem: Given the existence of a solution of (2.2) defined on $\left(0, r^{*}\right)$ with a singularity at $r=r^{*}$, satisfying $f(r)>0$ and $f^{\prime}(r)>0$ for $r \in\left(0, r^{*}\right)$, and having the asymptotic behaviour (a) in Proposition (5.3) as $r \rightarrow 0$, determine the dependence of $r^{*}$ on the parameters $f_{0}>0, a_{0}>0$ in the asymptotic expansion (4.4).

Similarly one may formulate analogous connection problems for the other cases $(b)-(d)$ in Proposition (5.3). The reason why we focus particularly on case $(a)$ is that in that case the Hessian matrix $\mathbf{H}$ is positive definite and $\operatorname{det} \mathbf{H}(r)$ remains positive and finite in the limit $r \rightarrow 0$. Thus a solution of the ODE (2.2) of type (a) yields a metric (1.2) on the interior of a ball of radius $r^{*}$ in $\mathbb{R}^{n}$. Also note that, because the local expansion (4.4) depends on two arbitrary constants $f_{0}, a_{0}$, type $(a)$ corresponds to the general solution of this second order ODE. In the next section we prove the existence of a type $(a)$ solution, by a direct construction using a nonlinear integral equation. The corresponding connection problem seems more difficult, but one possible approach would be to consider the inverse function $r(f)$. It is interesting to observe that the differential equation for the inverse function has an almost identical form to (2.2), namely

$$
\frac{d^{2} r}{d f^{2}}=-\frac{(n-1)}{r}\left(\frac{d r}{d f}\right)^{2}+\left(\frac{(n-1)}{f}-Q(r)\right) \frac{d r}{d f} .
$$

The leading order asymptotics (4.15) in the neighbourhood of the logarithmic branch point at $r=r^{*}$ extends to a corresponding double asymptotic series

$$
r(f) \sim r^{*}+\sum_{j=1}^{\infty} S_{j}(f) e^{-j Q\left(r^{*}\right) f}, \quad S_{1}(f)=-1+o(1), \quad f \rightarrow \infty
$$

in the equation (5.2); each of the $S_{j}$ is a series in powers of $f^{-1}$.

6. Solution of a nonlinear integral equation. Although the origin is a singular point of the ODE (2.2), it is possible to construct the general solution which is analytic on an interval $(0, R)$, with the behaviour (4.4) as $r \rightarrow 0$, by converting it to a nonlinear integral equation and then applying a variety of Picard iteration using Lipschitz type arguments. The integral equation produces the solution on a fixed interval, and then its analytic continuation must have a singularity at some $r^{*}>R$ by the results of the previous section. The key to the integral equation is in expressing the ODE in the form (2.5), which leads to the following

Proposition 6.1. For any fixed $r_{1}, r_{2}$ and arbitrary constants $C_{1}, C_{2}$, the ODE (2.2) is equivalent to the nonlinear integral equation

$$
f(r)=\left(C_{1} \int_{r_{2}}^{r} t^{n-1} e^{\Psi[f](t)} d t+C_{2}\right)^{1 / n},
$$

where

$$
\Psi[f](t):=\left(\frac{\kappa}{n} t-\frac{\lambda}{t^{n-1}}\right) f(t)-\int_{r_{1}}^{t}\left(\frac{\kappa}{n}+(n-1) \frac{\lambda}{s^{n}}\right) f(s) d s .
$$

Proof. Using integration by parts followed by exponentiation, the expression (2.5) yields immediately

$$
n f^{n-1} f^{\prime}=C_{1} r^{n-1} e^{\Psi[f](r)},
$$


for arbitrary constant $C_{1}$, with the endpoint of integration $r_{1}$ fixed as in (6.2). But the left hand side above is just the derivative of $f^{n}$, so after integrating from the point $r_{2}$, with constant of integration $C_{2}$, and taking the $n$th root, (6.1) results.

Observe that that for a fixed choice of $r_{1}, r_{2}$, the two constants $C_{1}, C_{2}$ represent the integration constants in the general solution of the ODE. Alternatively $r_{1}$ and $r_{2}$ can be allowed to vary, so that (6.1) has the form of a nonlinear variation of parameters formula. We remark that nonlinear integral equations like (6.1) have been used in [22] to analyse the solutions of Painlevé equations in the neighbourhood of movable pole singularities. The monograph by Sachdev [33] includes numerous further examples and references on integral equations applied to the asymptotics of ODEs. Iteration of nonlinear integral operators has also been used to prove existence of solutions to differential equations with proscribed asymptotics around an irregular singular point at infinity [35]. However, here we will employ the integral equation to construct the general solution of the ODE (2.2) on an interval of the real line starting at the fixed singularity at $r=0$, concentrating on the case of solutions that are positive and monotone increasing near the origin. To do so we construct a sequence of functions that successively approximate the solution with asymptotics (4.4) for $f_{0}>0, a_{0}=f_{0} \delta>0$.

Proposition 6.2. Given the positive constant $f_{0}>0$, the recurrence

$$
f_{j+1}(r)=f_{0}\left(1+n^{2} \delta \int_{0}^{r} t^{n-1} e^{\psi\left[f_{j}\right](t)} d t\right)^{1 / n}, \quad j=0,1,2, \ldots
$$

with

$$
\psi[f](t):=\int_{0}^{t}\left(\frac{\kappa}{n} s-\frac{\lambda}{s^{n-1}}\right) f^{\prime}(s) d s,
$$

and $\delta>0$ being another positive constant, defines a sequence of $C^{1}$ functions on the interval $[0, R]$. The endpoint of the interval $R>0$ is chosen to satisfy

$$
\frac{\kappa}{n(n+1)} R^{n+1}+|\lambda| R=\tilde{M}^{-1}, \quad \tilde{M}=f_{0} n \delta e,
$$

and the functions $f_{j}$ and their derivatives are bounded by

$$
f_{0} \leq f_{j}(r) \leq f_{0}\left(1+n \delta e r^{n}\right)^{1 / n}, \quad 0 \leq f_{j}^{\prime}(r) \leq \tilde{M} r^{n-1}, \quad r \in[0, R] .
$$

Proof. The bounds (6.6) are trivially true when $j=0$, and the proof proceeds by induction. Note that for $j=1$ we have

$$
f_{1}(r)=f_{0}\left(1+n \delta r^{n}\right)^{1 / n}
$$

corresponding to the special solution $(2.7)$, which is analytic on $[0, R]$. Now by induction

$$
\begin{gathered}
\left|\psi\left[f_{j}\right](r)\right| \leq \int_{0}^{r}\left(\frac{\kappa}{n} s+\frac{|\lambda|}{s^{n-1}}\right)\left|f_{j}^{\prime}(s)\right| d s \leq \int_{0}^{r}\left(\frac{\kappa}{n} s+\frac{|\lambda|}{s^{n-1}}\right) \tilde{M} s^{n-1} d s \\
=\tilde{M}\left(\frac{\kappa}{n(n+1)} r^{n+1}+|\lambda| r\right) \leq \tilde{M}\left(\frac{\kappa}{n(n+1)} R^{n+1}+|\lambda| R\right)=1,
\end{gathered}
$$


with the upper bound $R$ chosen as the unique positive real root of the equation (6.5). It follows that $\psi\left[f_{j}\right](r)$ exists and is continuous with $\psi\left[f_{j}\right](0)=0$. Defining

$$
X_{j}(r)=n^{2} \delta \int_{0}^{r} t^{n-1} e^{\psi\left[f_{j}\right](t)} d t
$$

it is clear that $X_{j}$ is also continuously differentiable and by (6.8) satisfies

$$
0 \leq X_{j}(r) \leq n^{2} \delta e \int_{0}^{r} t^{n-1} d t=n \delta e r^{n}
$$

for $r \in[0, R]$, with $X_{j}(0)=0$, so that $f_{j+1}(0)=f_{0}$, and (by monotonicity of $(1+X)^{1 / n}$ in $X$ ) the first bound (6.6) is satisfied by $f_{j+1}=f_{0}\left(1+X_{j}\right)^{1 / n}$. Furthermore (6.3) may be differentiated to give

$$
0 \leq f_{j+1}^{\prime}=f_{0} n \delta\left(1+X_{j}\right)^{\frac{1}{n}-1} r^{n-1} e^{\psi\left[f_{j}\right]} \leq f_{0} n \delta e^{\left|\psi\left[f_{j}\right]\right|} r^{n-1},
$$

and this derivative is continuous with $f_{j+1}^{\prime}(r) \leq f_{0} n \delta e r^{n-1}=\tilde{M} r^{n-1}$ as required, which completes the induction.

Remark. We can further assert that the functions $f_{j}$ are analytic on the interval, with Taylor series at the origin given by

$$
f_{j}(r)=f_{0}+f_{0} \delta r^{n}+O\left(r^{n+1}\right), \quad j=1,2, \ldots,
$$

since from (6.3) they are defined recursively by successive integrations of analytic functions starting from the constant function $f_{0}$. However, this is not needed for what follows.

TheOrem 6.3. The sequence of functions $\left\{f_{j}(r)\right\}_{j=0}^{\infty}$ defined on $[0, R]$ by the recurrence (6.3) as in Proposition 6.2 converges uniformly on $[0, R)$ to the function $f_{\infty}(r)$ which is a solution of the ODE (2.2) on this interval (except for the singular point $r=0$ ).

Proof. We take the inductive hypothesis

$$
\left|f_{j+1}(r)-f_{j}(r)\right| \leq f_{0} \delta \frac{r^{n+j}}{R^{j}}
$$

for $r \in[0, R]$. By the Mean Value Theorem

$$
(1+X)^{1 / n}-(1+Y)^{1 / n}=(1+Z)^{1 / n-1}(X-Y) / n
$$

for some $Z \in(X, Y)$, which gives

$$
\left|f_{1}-f_{0}\right|=f_{0}\left|\left(1+n \delta r^{n}\right)^{1 / n}-1\right| \leq f_{0} \delta r^{n}
$$

as required, and using the notation (6.9) this also implies

$$
\begin{aligned}
\left|f_{j+2}-f_{j+1}\right| & =f_{0}\left|\left(1+X_{j+1}\right)^{1 / n}-\left(1+X_{j}\right)^{1 / n}\right| \\
& \leq f_{0} n^{-1}\left|X_{j+1}-X_{j}\right| \\
& =f_{0} n \delta\left|\int_{0}^{r} t^{n-1}\left(e^{\psi\left[f_{j+1}\right](t)}-e^{\psi\left[f_{j}\right](t)}\right) d t\right|,
\end{aligned}
$$


since $X_{j} \geq 0$ for all $j$ as in the proof of Proposition 6.2. Thus we have

$$
\begin{aligned}
\left|f_{j+2}-f_{j+1}\right| & \leq f_{0} n \delta \int_{0}^{r} t^{n-1}\left|e^{\psi\left[f_{j+1}\right](t)}-e^{\psi\left[f_{j}\right](t)}\right| d t \\
& \leq f_{0} n \delta e \int_{0}^{r} t^{n-1}\left|\psi\left[f_{j+1}\right](t)-\psi\left[f_{j}\right](t)\right| d t \\
& =\tilde{M} \int_{0}^{r} t^{n-1}\left|\int_{0}^{t}\left(\frac{\kappa}{n} s-\frac{\lambda}{s^{n-1}}\right)\left(f_{j+1}^{\prime}(s)-f_{j}^{\prime}(s)\right) d s\right| d t,
\end{aligned}
$$

where the Mean Value Theorem has been used once more to bound the difference of the two exponentials, together with the fact that $\left|\exp \psi\left[f_{j}\right](r)\right| \leq e$ for all $j$ by (6.8). Integrating by parts and using the fact that $\left|f_{j+1}(s)-f_{j}(s)\right|=O\left(s^{n+j}\right)$ to evaluate the boundary term at $s=0$, then further using the inductive hypothesis (6.11) to bound each of the terms that follow results in

$$
\begin{aligned}
& \left|\int_{0}^{t}\left(\frac{\kappa}{n} s-\frac{\lambda}{s^{n-1}}\right)\left(f_{j+1}^{\prime}(s)-f_{j}^{\prime}(s)\right) d s\right| \\
& =\left|\left(\frac{\kappa}{n} t-\frac{\lambda}{t^{n-1}}\right)\left(f_{j+1}(t)-f_{j}(t)\right)-\int_{0}^{t}\left(\frac{\kappa}{n}+\frac{(n-1) \lambda}{s^{n}}\right)\left(f_{j+1}(s)-f_{j}(s)\right) d s\right| \\
& \leq f_{0} \delta R^{-j}\left(\kappa n^{-1} t^{n+j+1}+|\lambda| t^{j+1}+\int_{0}^{t}\left(\kappa n^{-1} s^{n+j}+(n-1)|\lambda| s^{j}\right) d s\right) \\
& =f_{0} \delta R^{-j}\left(\frac{\kappa(n+j+2)}{n(n+j+1)} t^{n+j+1}+\frac{|\lambda|(n+j)}{(j+1)} t^{j+1}\right) .
\end{aligned}
$$

Substituting into the previous bound and integrating yields

$$
\begin{aligned}
\left|f_{j+2}-f_{j+1}\right| & \leq \tilde{M} f_{0} \delta R^{-j}\left(\frac{\kappa(n+j+2)}{n(n+j+1)(2 n+j+1)} r^{2 n+j+1}+\frac{|\lambda|(n+j)}{(j+1)(n+j+1)} r^{n+j+1}\right) \\
& \leq \tilde{M} f_{0} \delta R^{-j}\left(\frac{\kappa}{n(n+1)} R^{n}+|\lambda|\right) r^{n+j+1}=f_{0} \delta r^{n+j+1} R^{-(j+1)}
\end{aligned}
$$

using (6.5), and the hypothesis is proved. Hence on any interval $0 \leq r \leq R^{*}<R$ the series

$$
\sum_{j=0}^{\infty}\left|f_{j+1}(r)-f_{j}(r)\right| \leq \sum_{j=0}^{\infty} f_{0} \delta \frac{r^{n+j}}{R^{j}}=f_{0} \delta r^{n}\left(1-\frac{r}{R}\right)^{-1} \leq f_{0} \delta\left(R^{*}\right)^{n}\left(1-\frac{R^{*}}{R}\right)^{-1}
$$

converges uniformly by the Weierstrass M-test. Also each of the functions $f_{j}$ is positive with non-negative derivative bounded by (6.6). It follows that the sequence of functions converges uniformly on $[0, R)$ to a function $f_{\infty}(r)$ satisfying $f_{\infty}(r) \leq f_{0}+f_{0} \delta r^{n}(1-r / R)^{-1}$. Similarly, using the inequalities

$$
\begin{aligned}
\left|f_{j+1}^{\prime}(r)-f_{j}^{\prime}(r)\right| & =f_{0} n \delta r^{n-1}\left|\left(1+X_{j}\right)^{1 / n-1} e^{\psi\left[f_{j}\right]}-\left(1+X_{j-1}\right)^{1 / n-1} e^{\psi\left[f_{j-1}\right]}\right| \\
\leq & f_{0} n \delta r^{n-1}\left(e^{\psi\left[f_{j}\right]}\left|\left(1+X_{j}\right)^{1 / n-1}-\left(1+X_{j-1}\right)^{1 / n-1}\right|+\right. \\
& \left.\quad+\left(1+X_{j-1}\right)^{1 / n-1}\left|e^{\psi\left[f_{j}\right]}-e^{\psi\left[f_{j-1}\right]}\right|\right) \\
& \leq f_{0} n \delta e r^{n-1}\left((1-1 / n)\left|X_{j}-X_{j-1}\right|+\left|\psi\left[f_{j}\right]-\psi\left[f_{j-1}\right]\right|\right) \\
& \leq \delta r^{n-1}\left(\tilde{M}(n-1) r^{n}+f_{0}(n+j+1)\right)(r / R)^{j}
\end{aligned}
$$


it is straightforward to prove that the sequence of derivatives $f_{j}^{\prime}$ converges uniformly. The Weierstrass M-test is satisfied by bounding the sum of the two terms in (6.13) with a geometric series and derivative of a geometric series respectively, and by standard theorems (such as Theorem 8.1 in [13]) it follows that the sequence $\left\{f_{j}^{\prime}\right\}_{j=0}^{\infty}$ has as its limit the derivative $f_{\infty}^{\prime}$. From (6.6) the limit function and its derivative satisfy the bounds

$$
f_{0} \leq f_{\infty}(r) \leq f_{0}\left(1+n \delta e r^{n}\right)^{1 / n}, \quad 0 \leq f_{\infty}^{\prime}(r) \leq \tilde{M} r^{n-1}
$$

Clearly $f_{\infty}(0)=\lim _{j \rightarrow \infty} f_{j}(0)=f_{0}$, and $f_{\infty}$ is monotone increasing. Furthermore, note that the inequality (6.13) also implies that the sequence of functions $f_{j}^{\prime}(r) / r^{n-1}$ converges uniformly, and thus there is a sequence of constants $\epsilon_{j}$ such that

$$
\frac{\left|f_{\infty}^{\prime}(r)-f_{j}(r)\right|}{r^{n-1}} \leq \epsilon_{j} \rightarrow 0 \quad \text { as } \quad j \rightarrow \infty
$$

Hence

$$
\begin{aligned}
\left|X_{\infty}-X_{j+1}\right| & \leq n^{2} \delta \int_{0}^{r} t^{n-1}\left|e^{\psi\left[f_{\infty}\right]}-e^{\psi\left[f_{j}\right]}\right| d t \leq n^{2} \delta e \int_{0}^{r} t^{n-1}\left|\psi\left[f_{\infty}\right]-\psi\left[f_{j}\right]\right| d t \\
& \leq n^{2} \delta e \int_{0}^{r} t^{n-1}\left|\int_{0}^{t}\left(\frac{\kappa}{n} s-\frac{\lambda}{s^{n-1}}\right)\left(f_{\infty}^{\prime}(s)-f_{j}^{\prime}(s)\right) d s\right| d t \\
& \leq n^{2} \delta e \int_{0}^{r} t^{n-1}\left(\int_{0}^{t}\left(\frac{\kappa}{n} s^{n}+|\lambda|\right) \epsilon_{j} d s\right) d t \rightarrow 0 \text { as } j \rightarrow \infty .
\end{aligned}
$$

Then taking the limit $j \rightarrow \infty$ in the right hand side of (6.3) we see that the function $f_{\infty}$ satisfies the integral equation

$$
f_{\infty}(r)=f_{0}\left(1+n^{2} \delta \int_{0}^{r} t^{n-1} e^{\psi\left[f_{\infty}\right](t)} d t\right)^{1 / n} \equiv f_{0}\left(1+X_{\infty}\right)^{1 / n}
$$

It follows immediately that $f_{\infty}$ is a solution of the ODE (2.2), upon differentiating (6.15); this is just a reversal of the steps of the proof of Proposition 6.1. This completes the proof of the Theorem. $\square$

Corollary 6.4. The ODE (2.2) has a solution of the type (a) in Proposition 5.3, given by the function $f_{\infty}(r)$ from Theorem 6.3 on the interval $(0, R)$. This solution has the asymptotic behaviour (4.4) as $r \rightarrow 0$ with parameter $a_{0}=f_{0} \delta$, and it has an analytic continuation along the real axis for $R \leq r<r^{*}$ with a singularity at some point $r=r^{*}$.

Proof. From the Theorem and its proof, the function $f_{\infty}(r)$ is a solution of the $\mathrm{ODE}$ on $(0, R)$ with the limit value $f_{\infty}(0)=f_{0}$, which is the first term in the asymptotic series (4.4), and by Proposition 3.1 the solution is analytic on the open interval. The limit as $r$ tends to zero from the right can be seen directly from the fact that it satisfies the integral equation (6.15), with the limits $X_{\infty}(r) \rightarrow 0, \psi\left[f_{\infty}\right](r) \rightarrow 0$ as $r \rightarrow 0+$. Differentiating (6.15) and using l'Hôpital's rule gives

$$
\begin{aligned}
\lim _{r \rightarrow 0+} & \frac{\left(f_{\infty}(r)-f_{0}-f_{0} \delta r^{n}\right)}{r^{n}}=\lim _{r \rightarrow 0+} \frac{\left(f_{\infty}^{\prime}(r)-f_{0} \delta n r^{n-1}\right)}{n r^{n-1}} \\
& =\lim _{r \rightarrow 0+} f_{0} \delta\left(\frac{e^{\psi\left[f_{\infty}\right](r)}}{\left(1+X_{\infty}(r)\right)^{1-1 / n}}-1\right)=0,
\end{aligned}
$$

which gives the second nonzero term in (4.4), with the coefficient of $r^{n}$ being $a_{0}=f_{0} \delta$. It is clear that $f_{\infty}(r)$ is $C^{\infty}$ at the endpoint $r=0$, by repeated differentiation of (6.15), 
since all the right limits exist as $r \rightarrow 0+$, and thus the terms in the series (4.4) can be obtained recursively from the integral equation. Given that derivatives of all orders exist, this is just the Taylor series at the endpoint $r=0$. Although the function $f_{\infty}$ has been constructed as the limit of a uniformly convergent sequence upper bounded by a geometric series (6.12) that diverges at $r=R$, the bounds (6.14) imply that the limit values $f_{\infty}(R)$ and $f_{\infty}^{\prime}(R)$ must be finite. Thus by Proposition 3.1, taking these limits as initial data at the point $\eta=R$, the solution of the ODE can be analytically continued to $r>R$, up to a minimum distance $\rho$ given by (3.3). Then by Proposition 5.1 it must reach a singularity at a point $r^{*} \geq R+\rho$.

Remark. Due to the remark by one of the referees, that setting $f(r)=f_{0}+r^{n} \psi(r)$ leads to a regular singularity in $\psi(r)$ at $r=0$, further results on asymptotic series (see e.g. $[37,35]$ ) mean that the solution of the ODE can be analytically continued off the positive semi-axis to a disc around $r=0$, in which case this is just a removable singularity and (4.4) acquires the status of a convergent Taylor series. It would be interesting to attempt to bound this series by direct estimates.

7. Conclusions. For any dimension $n>1$, new solutions of Abreu's equation (1.7) have been found by making a similarity reduction to $O(n)$ invariant solutions. The similarity solutions are determined in terms of a single function $f(r)$ depending only on the radial coordinate $r$, which satisfies a second order ODE (2.2) including an arbitrary parameter $\lambda$. Due to a singularity in the ODE at the origin, a standard initial value problem is not defined at $r=0$. Nevertheless we have shown that there is a class of solutions, with $f(r)$ being positive and monotone increasing, that exist on some interval $\left(0, r^{*}\right)$, having a finite limit as $r \rightarrow 0+$ and a singularity at $r=r^{*}$. For these solutions, the Hessian $\mathbf{H}(r)$ is positive definite on this interval, and its matrix elements $u_{j k}$ (1.3) define a metric (1.2) on the interior of a ball of radius $r^{*}$ in $\mathbb{R}^{n}$. Although this is not specifically relevant to the case of toric varieties, where Abreu's equation (1.7) is solved on a polytope $P$, it fits within the more general setting outlined in [11], with the Mabuchi functional $\mathcal{F}$ (1.8) defined on a bounded open convex subset of $\mathbb{R}^{n}$. This raises the question of whether a rotation invariant solution might be a minimizer of $\mathcal{F}$ defined on the ball. However, whether the corresponding metric (1.2) on the interior of the ball could be extended to a metric on a $2 n$-dimensional symplectic manifold is uncertain.

It would also be interesting to find an analogue of Theorem 6.3 for consideration of the other asymptotic behaviours at the origin, i.e. cases (b) and (c) in Proposition 4.1. However, in that case the Hessian and inverse Hessian of $u$ would no longer both be finite at $r=0$.

There are many remarkable connections between integrable systems and differential geometry [7], going back to classical work of Darboux and Bianchi on the geometry of surfaces. There is considerable evidence [2] that the concept of integrability is strongly related to the Painlevé property, that the general solution of an equation should have only poles as movable singularities. Recently the Painlevé property (or a weaker version thereof) has been used to derive explicit metrics for relativistic fluids [15]. The various Painlevé tests are good heuristic tools for analysing ordinary and partial differential equations, and have been used to isolate new integrable systems (see chapter 7 of [3] for a review).

The motivation behind the preceding analysis was to see whether the elegant fourth order PDE (1.7) might be integrable, by examining the singularity structure of the similarity reduction in the light of the Ablowitz-Ramani-Segur conjecture [2]. The ODE (2.2) has not only algebraic branching (4.14) but also movable logarithmic 
branch points (4.15), and so certainly fails the standard Painlevé test or its weak extension [32]. This would seem to indicate that Abreu's equation (1.7) is not integrable. However, as originally emphasized by the authors of [2], the Painlevé property is extremely sensitive to changes of variables. For example, transformations of hodograph type can change equations with movable algebraic branch points into equations that have only poles $[9,18]$. It has also been observed [31] that when an equation is written in potential form it can have a single (or finitely many) logarithmic terms in a local expansion around singular points, and still be integrable.

In the case of the KP equation (1.10), the potential $w$ is given by the logarithmic derivative of the tau-function:

$$
w=-\frac{\partial}{\partial x} \log \tau
$$

The tau-function for the rational solutions (1.11) takes the form

$$
\tau=\prod_{l=1}^{d}\left(x-x_{l}(\underline{t})\right),
$$

and this is a polynomial both in $x$ and the times $\underline{t}$. For the more general algebrogeometric solutions of KP [24], the tau-function is a theta-function of an arbitrary Riemann surface. The rational and soliton solutions arise as degenerate limits of the theta-functions. It would be interesting to see if Abreu's equation could admit quasiperiodic generalizations of (1.9), by dropping the extremality condition (1.5) on the the curvature, allowing $S$ in (1.1) to be a (non-constant) periodic function.

Other methods of testing for integrability might provide useful information about Abreu's equation. Since (1.7) is not of evolution type, a promising method would be the symmetry approach of Shabat et al [27], which has recently been extended [28] in order to deal with non-evolutionary equations. It would also be interesting to apply the methods of [30] to look for other sorts of group invariant solutions of Abreu's equation.

Acknowledgments. I am grateful to Simon Donaldson for useful comments and encouragement, and for making the preprint of [11] available. I would like to thank the London Mathematical Society for supporting my attendance at the Mary Cartwright meeting. Also thanks to Alexander Tovbis for providing a copy of [35]. Finally, I am grateful to the referees for helpful remarks and suggestions.

\section{REFERENCES}

[1] S. Abenda And Y. Fedorov, On the weak Kowalevski-Painlevé property for hyperelliptically separable systems, Acta Appl. Math., 60 (2000), pp. 137-178.

[2] M.J. Ablowitz, A. Ramani and H. Segur, Nonlinear evolution equations and ordinary differential equations of Painlevé type, Lett. Nuovo Cim., 23 (1978), pp. 333-338.

[3] M.J. Ablowitz and P.A. Clarkson, Solitons, Nonlinear Evolution Equations and Inverse Scattering, Cambridge University Press (1991).

[4] M. Abreu, Kähler geometry of toric varieties and extremal metrics, Internat. J. Math., 9 (1998), pp. 641-651.

[5] M. Abreu, Kähler metrics on toric orbifolds, J. Diff. Geom., 58 (2001), pp. 151-187.

[6] M. Bennai and E.H. Saidi, Toric varieties with NC toric actions: NC type IIA geometry, Nucl. Phys. B, 677 (2004), pp. 587-613.

[7] A.I. Bobenko and R. Seiler (EDS.), Discrete Integrable Geometry and Physics, Oxford University Press (1999). 
[8] J. Chazy, Acta Math., Sur les équations différentielles du troisième ordre et d'ordre supérieur dont l'intégrale générale a ses points critiques fixes, 34 (1911), pp. 317.

[9] P.A. Clarkson, A.S. Fokas and M.J. Ablowitz, Hodograph Transformations on Linearizable Partial Differential Equations, SIAM J. Appl. Math., 49 (1989), pp. 1188-1209.

[10] D. Cox, What is a Toric Variety? in Proc. Workshop on Geometric Modeling and Algebraic Geometry, Vilnius, Lithuania, August 2003; http://www.cs.amherst.edu/ dac/lectures/tutorial.ps

[11] S.K. Donaldson, Convex analysis and toric manifolds, lecture at LMS Mary Cartwright meeting (2002); Scalar curvature and stability of toric varieties, Jour. Diff. Geom., 62 (2002), pp. 289-349.

[12] S.K. DonaldSon, private communication (2003).

[13] E. Fischer, Intermediate Real Analysis, Springer-Verlag (1983).

[14] V. Guillemin, Kaehler structures on toric varieties, J. Diff. Geom., 40 (1984), pp. 285-309.

[15] R.G. Halburd, Shear-free Relativistic Fluids and the Absence of Movable Branch Points, J. Math. Phys., 43 (2002), pp. 1966-1979.

[16] E. Hille, Ordinary Differential Equations in the Complex Domain, Wiley, New York (1976).

[17] A. Hinkkanen and I. Laine, Solutions of the first and second Painlevé equations are meromorphic, J. Analyse Math., 79 (1999), pp. 345-377.

[18] A.N.W. Hone, Reciprocal transformations, Painlevé property and exact solutions of energydependent Schrödinger hierarchies, Phys. Lett. A, 249 (1998), pp. 46-54.

[19] A.N.W. Hone, $O(n)$ invariant solutions of Abreu's equation, math.DG/0209123.

[20] A.N.W. Hone, Ordinary differential equations, nonlinear, in Encyclopedia of Nonlinear Science, A. Scott (Ed.), Routledge (2004) in press; http://www.routledge-ny.com/nonlinearsci

[21] E.L. Ince, Ordinary Differential Equations (1926). Reprint: New York: Dover Publications (1956).

[22] N. Joshi And M.D. Kruskal, A Direct Proof that Solutions of the Six Painleve Equations have no Movable Singularities Except Poles, Stud. Appl. Math., 93 (1994), pp. 187.

[23] M.D. Kruskal, N. Joshi and R. Halburd, Analytic and Asymptotic Methods for Nonlinear Singularity Analysis: a Review and Extensions of Tests for the Painlevé Property, Proceedings of CIMPA Summer School on Nonlinear Systems, eds. B. Grammaticos and K. Tamizhmani, Lecture Notes in Physics 495, Springer-Verlag, Heidelberg (1997) 171-205.

[24] I.M. KRICheVER, Methods of algebraic geometry in the theory of non-linear equations, Russian Math. Surveys, 32 (1977), pp. 185-213.

[25] I.M. Krichever, About rational solutions of the Kadomtsev-Petviashvili equation and integrable systems of particles on the line, Funct. Anal. Appl., 12 (1978), pp. 59-61.

[26] T. Mabuchi, K-energy maps integrating Futaki invariants, Tohoku Math. Jour., 38 (1986), pp. $575-593$.

[27] A.V. Mikhailov, A.B. Shabat and R.I. Yamilov, The Symmetry Approach to the Classification of Non-linear Equations. Complete Lists of Integrable Systems, Russian Math. Surveys, 42 (1987), pp. 1-63.

[28] A.V. Mikhailov and V S. Novikov, Perturbative symmetry approach, J. Phys. A: Math. Gen., 35 (2002), pp. $4775-4790$.

[29] Y. Ohta, J. Satsuma, D. Takahashi and T. Tokiniro, An Elementary Introduction to Sato Theory, Prog. Theor. Phys. Suppl., 94 (1988), pp. 210-241.

[30] P.J. Olver, Applications of Lie Groups to Differential Equations, 2nd Edition, Springer-Verlag (1993).

[31] A. Pickering, The singular manifold method revisited, J. Math. Phys., 37 (1996), pp. 18941927.

[32] A. Ramani, B. Dorizzi and B. Grammaticos, Painlevé conjecture revisited, Phys. Rev. Lett., 49 (1982), pp. $1538-1541$.

[33] P.L. SACHDEv, Nonlinear ordinary differential equations and their applications, Marcel Dekker, New York (1991).

[34] T. Shiota, Calogero-Moser hierarchy and KP hierarchy, J. Math. Phys., 35 (1995), pp. 58445849 .

[35] A. TovBis, Nonlinear Ordinary Differential Equations Resolvable with Respect to an Irregular Singular Point, J. Diff. Eq., 109 (1994), pp. 201-221.

[36] A.P. Veselov, On geometry of a special class of solutions to generalised WDVV equations, in Integrability: The Seiberg-Witten and Whitham equations, eds. H. Braden and I. Krichever, Gordon and Breach (2000), pp. 125-136.

[37] W. Wasow, Asymptotic Expansions for Ordinary Differential Equations, Wiley, New York (1965). 